Article

\title{
Investigation of Oriented Magnetic Field Effects on Entropy Generation in an Inclined Channel Filled with Ferrofluids ${ }^{+}$
}

\author{
Elgiz Baskaya ${ }^{1}$, Guven Komurgoz ${ }^{2, *}$ and Ibrahim Ozkol ${ }^{3}$ \\ Ecole Nationale de l'Aviation Civile, 31400 Toulouse, France; elgiz.baskaya@enac.fr \\ Faculty of Electrics \& Electronics, Istanbul Technical University, 34469 Istanbul, Turkey \\ 3 Faculty of Aeronautics \& Astronautics, Istanbul Technical University, 34469 Istanbul, Turkey; \\ ozkol@itu.edu.tr \\ * Correspondence: komurgoz@itu.edu.tr; Tel.: +90-549-745-3713 \\ + This paper is an extended version of our paper published in ASME 2014 12th Biennial Conference on \\ Engineering Systems Design and Analysis, Copenhagen, Denmark, 25-27 July 2014.
}

Received: 13 June 2017; Accepted: 18 July 2017; Published: 23 July 2017

\begin{abstract}
Dispersion of super-paramagnetic nanoparticles in nonmagnetic carrier fluids, known as ferrofluids, offers the advantages of tunable thermo-physical properties and eliminate the need for moving parts to induce flow. This study investigates ferrofluid flow characteristics in an inclined channel under inclined magnetic field and constant pressure gradient. The ferrofluid considered in this work is comprised of $\mathrm{Cu}$ particles as the nanoparticles and water as the base fluid. The governing differential equations including viscous dissipation are non-dimensionalised and discretized with Generalized Differential Quadrature Method. The resulting algebraic set of equations are solved via Newton-Raphson Method. The work done here contributes to the literature by searching the effects of magnetic field angle and channel inclination separately on the entropy generation of the ferrofluid filled inclined channel system in order to achieve best design parameter values so called entropy generation minimization is implemented. Furthermore, the effect of magnetic field, inclination angle of the channel and volume fraction of nanoparticles on velocity and temperature profiles are examined and represented by figures to give a thorough understanding of the system behavior.
\end{abstract}

Keywords: ferrofluid; inclined channel; magnetic field; Generalized Differential Quadrature Method

\section{Introduction}

Currently, the cooling needs of cutting edge technologies pose a challenge to existing cooling fluids since they are actually poor conductors of heat. The chance of designing an enhanced, more conductive cooling fluid led some researchers to discover the strange world of the nano size. Nanofluids, engineered colloidal suspensions of nanoparticles (typically less than $100 \mathrm{~nm}$ ) in a base fluid, usually conventional cooling fluids, seem to be a new key to hurdle with the thermal bottleneck for various applications. Thus the research guided to these tiny particles as efficient tools to cope up with the thermal needs of not only small in size applications such as microelectromechanical systems (MEMS), but also giant processes such as nuclear reactors. The underlying mechanisms of the novel properties concerning nanofluids are still a mystery from the scientific point of view. That is the reason for many researchers being amazed by the capability achieved by just adding very tiny particles, typically made of metals, oxides, carbides or carbon nanotubes into conventional cooling fluids. New models proposed, many experiments initiated to understand the new phenomenon.

The idea to utilize particles to enhance convective heat transfer and thermal conductivity goes back to Maxwell [1], when he used micro particles within a base fluid. Back then, the method did not 
work well due to constraints mostly inherent to the size of the particles and the experiments resulted unsatisfactory due to clogging, erosion, rapid sedimentation and high-pressure drop. Advancement of technology enabled to explore the world of tinier particles. Coined the name "nanofluids" by Choi [2], this innovative engineering fluid gained popularity with same author's work showing an evident increase in thermal conductivity is achieved by using nanofluids instead of a conventional heat transfer fluid. Research community immediately directed to this newer topic since heat transfer is a game changer in most of the engineering designs and various work dedicated to show superiority of their thermal conductivity [3-7]. Investigations on not only the heat transfer properties but also tribology shows nanoparticle addition to lubricating oils improves the load-carrying, and friction reduction features [8,9]. Eastman et al. [10] showed that the thermal conductivity increased up to $40 \%$ compared to its basefluid by using copper nanoparticles in ethylene glycol with a solid volume ratio of $0.3 \%$. Various geometries have been investigated such as trapezoidal cavities [11], porous cavities [12], and channels [13]. Nanofluid flow has been also deeply studied as a function of different parameters such as volume fraction and nanoparticle size for a variety of models offered [14]. But the applications are not yet constrained by the engineering community, the interest of the medical domain reached at a level not to be underestimated. Drug targeting is an example of promising medical applications of nanofluids for cancer treatment [15]. Injected magnetic nanoparticles with chemotherapeutic agents could be directed to the tumour in order to cause less damage to the surrounding tissues with the utilization of an external magnetic field [16-20]. Enlarging sets of applications of these magnetic nanofluids caused them to be given an alies as ferrofluids. The ability to control the flow via an external magnetic field also raises the questions to have an optimal flow by decreasing the entropy generation. Such an idea drives to more research on entropy generation in the presence of nanofuids [21].

Designing systems optimally, drive the search for a measure of destruction of system's available work. Entropy generation can be imagined as a measure for the irreversibility associated to the processes. The idea of entropy generation goes back to 1824, when Carnot recognized the importance of avoiding irreversible processes since entropy is produced as a result [22]. Clasius was the one to introduce the term "entropy", and also gave a mathematical expression for entropy production [23,24]. Bejan presented the idea of entropy generation minimization in order to identify the factors responsible for the loss of available work of the system [25-30]. Since then, numerous studies have been conducted on entropy generation minimization to utilize energy efficiently under various flow conditions [31-34]. Analytical entropy generation analysis for modelling and optimization of magnetohydrodynamic induction devices is investigated by Salas et al. [35]. Mahmud et al. [36] studied thermodynamics analysis of mixed convection in a channel with transverse hydromagnetic effect. Chauhan and Kumar conducted a study on the heat transfer and entropy generation during compressible fluid flow in a channel partially filled with porous medium [37]. Entropy generation in a porous channel with hydromagnetic effects is investigated by Tasnim et al. [38]. Eegunjobi and Makinde [39] analysed the combined effect of buoyancy force and Navier slip on entropy generation in a vertical porous channel. Jery et al. presented the effect of an external oriented magnetic field on entropy generation in natural convection [40]. The incompressible viscous laminar flow through a channel filled with porous media is studied by Dwivedi et all [41]. Numerical investigation of buoyancy effects on hydromagnetic unsteady flow through a porous channel with suction/injection is conducted by Makinde and Chinyoka [42].

In this work, magnetic nanoparticles' effects on flow are studied with regard to changing magnetic field applied from outside the flow. Exhibiting both magnetic and fluid properties, magnetic nanofluids (ferrofluids) constitute a special class, with the flexibility to be controlled by an external magnetic field. By merging the science on nanofluids such as available models describing nanofluid properties and optimal system design with grounds on entropy generation minimization, effects magnetic field change on inclined channel flow has been studied. The magnetic field angle and channel inclination is handled separately, considering the cases for fixed inlined channels. To solve the equation set, a semi numerical tool, Generalized Differential Quadrature Method (GDQM), is used for discretization for its advantages such as computationally efficiency. GDQM is a numerical technique for solving differential 
equations by approximating the derivative of a function at any location by a linear summation of all the functional values along a mesh line. Then the resulting sets of algebraic equations are solved with Newton-Raphson (NR) method. The effect of magnetic field, and nanofluid variable properties on heat transfer enhancement and rheology are examined and represented by various figures to give a thorough understanding of the system efficiency behavior. This work contributes to the literature by showing the effects of magnetic field angle and channel inclination separately on the entropy generation of the ferrofluid filled inclined channel system to achieve an optimal design.

\section{Mathematical Modelling}

In this study, steady viscous incompressible flow of a Cu-Water nanofluid bounded by two infinite horizontal parallel plates with a distance $H$ in between is considered. Cartesian coordinate axes are selected as reference, $x$-axis parallel to plates and located at the same distance to the plates, $y$-axis along the direction perpendicular to the plates as can be seen in Figure 1. A constant magnetic field is applied perpendicular to the plates uniformly along $y$-axis. The plates are sustained to have a constant temperature of $T_{w_{2}}$ and $T_{w_{1}}$, at the lower and upper plates respectively. The assumptions carried through the study can be summarized as:

1. The plates are infinitely long, enabling to consider the problem one dimensional.

2. The induced magnetic field is neglected when compared with the applied magnetic field, due to inherently small magnetic Reynolds number for magnetic liquids and partially ionized fluids [43].

3. Electric field and Hall effects, the ion-slip and thermoelectric effects, and the electron pressure gradient are neglected [44-46].

4. The flow is assumed to be fully developed and the edge effects are neglected.

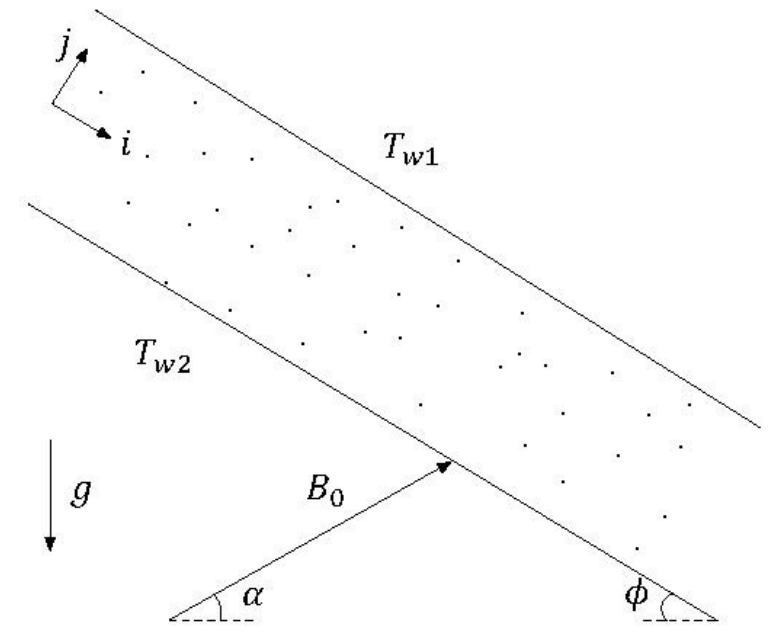

Figure 1. Schematic configuration of the studied problem.

Literature for an inclined channel problem is quite rich yet still requires to be combined to point the specific problem tackled. As such, in [47] discusses the influence of inclined magnetic field on peristaltic flow of a Williamson fluid model in an inclined channel without nanofluid. In [40] encounters the effects of and external oriented magnetic field on entropy in a cavity configuration without nanoparticles. Reference [48] studies the MHD effects on heat transfer and entropy generation of nanofluid flow in an open cavity in the presence of an inclined magnetic field. References $[49,50]$ also referred for their application of inclined channel flow in the presence of nanofluid with fixed external magnetic field. The missing effects of one another has been merged to find out the equations of motion which simplifies to the equations in the literature for under special conditions, such as constant external magnetic field. 
The schematic configuration of the considered system is given in Figure 1. The inclined channel filled with nanofluids is exposed to magnetic field $B_{0}$. Under these assumptions the nonlinear coupled differential equations governing the system can be written as Equations (1) and (2).

$$
\begin{aligned}
\mu_{n f} \frac{\partial^{2} u}{\partial y^{2}}+(\rho \beta)_{n f} g \sin \phi\left(T-T_{w 2}\right)-\sigma_{n f} B_{0}^{2} \sin ^{2}(\alpha+\phi) u & =\frac{\partial p}{\partial x} \\
\frac{\partial^{2} T}{\partial y^{2}}+\frac{\mu_{n f}}{k_{n f}}\left(\frac{\partial u}{\partial y}\right)^{2}+\frac{\sigma_{n f}}{k_{n f}} B_{0}^{2} \sin ^{2}(\alpha+\phi) u^{2} & =0
\end{aligned}
$$

The system of equations in Equations (1) and (2) is subject to boundary conditions Equations (3) and (4).

$$
\begin{array}{ll}
u(H / 2)=0 & u(-H / 2)=0 \\
T(H / 2)=T_{w 1} & T(-H / 2)=T_{w 2}
\end{array}
$$

Addition of nanoparticles changes the thermophysical properties of the fluid flow. There are numerous work in open literature to model the surprising nature of the nanofluids but there is no consensus yet. Available models depend on different parameters, e.g., the nanoparticle material, the volume fraction of nanofluids, specific heat, and others. In this study, thermal diffusivity of the nanofluid $\alpha_{n f}$ and effective density of the nanofluid $\rho_{n f}$ is expressed as.

$$
\begin{aligned}
\alpha_{n f} & =\frac{k_{n f}}{\left(\rho c_{p}\right)_{n f}} \\
\rho_{n f} & =(1-\psi) \rho_{f}+\psi \rho_{p}
\end{aligned}
$$

Effective dynamic viscosity of the nanofluid is given by the Brinkmann model [51] as Equation (7).

$$
\frac{\mu_{n f}}{\mu_{f}}=\frac{1}{(1-\psi)^{2.5}}
$$

The effective thermal conductivity $k_{n f}$ and the electrical conductivity $\sigma_{n f}$ of the nanofluid are modeled by Maxwell [1] and given as.

$$
\begin{aligned}
& \frac{k_{n f}}{k_{f}}=\frac{k_{p}+2 k_{f}-2 \psi\left(k_{f}-k_{p}\right)}{k_{p}+2 k_{f}+\psi\left(k_{f}-k_{p}\right)} \\
& \frac{\sigma_{n f}}{\sigma_{f}}=1+\frac{3\left(\frac{\sigma_{p}}{\sigma_{f}}-1\right) \psi}{\left(\frac{\sigma_{p}}{\sigma_{f}}+2\right)-\left(\frac{\sigma_{p}}{\sigma_{f}}-1\right) \psi}
\end{aligned}
$$

To derive the dimensionless forms of the governing equations, the following parameters in Equations (10)-(12) are defined.

$$
\begin{aligned}
& Y=\frac{y}{h} \\
& U=\frac{u H}{\alpha_{f}} \\
& P=\frac{H^{3}}{\alpha_{f}^{2} \rho_{n f}}\left(-\frac{\partial p}{\partial x}\right)
\end{aligned}
$$


Some other dimensionless parameters describing characteristics of the fluid flow utilized in dimensionalization can be listed as Equations (13)-(15).

$$
\begin{array}{rlrl}
H a & =B 0 H \sqrt{\frac{\sigma_{n f}}{\rho_{n f} v_{f}}} & R a=\frac{g \beta_{f} H^{3}\left(T_{w 2}-T_{w 1}\right)}{v_{f} \alpha_{f}} \\
E c=\frac{\alpha_{f}^{2}}{H^{2} C_{P_{f}}\left(T_{w 2}-T_{w 1}\right)} & \operatorname{Pr}=\frac{\mu_{f} C_{P_{f}}}{k_{f}} \\
B r=E c \cdot \operatorname{Pr}=\frac{\alpha_{f}^{2} \mu_{f}}{H^{2}\left(T_{w 2}-T_{w 1}\right) k_{f}} &
\end{array}
$$

Considering the dimensionless parameters, the non-dimensionalized differential equations describing the flow can be summarized with Equations (16) and (17).

$$
\begin{array}{r}
\frac{\mu_{n f}}{\rho_{n f} \alpha_{f}} \frac{\partial^{2} U}{\partial Y^{2}}+\frac{(\rho \beta)_{n f}}{\rho_{n f} \beta_{f}} \sin \phi R a \operatorname{Pr} \theta-\operatorname{Pr} H a^{2} \sin ^{2}(\alpha+\phi) U+P=0 \\
\frac{\partial^{2} \theta}{\partial Y^{2}}+\frac{k_{f} \mu_{n f}}{k_{n f} \mu_{f}} B r\left(\frac{\partial U}{\partial Y}\right)^{2}+\frac{\rho_{n f} k_{f}}{\rho_{f} k_{n f}} B r H a^{2} \sin ^{2}(\alpha+\phi) U^{2}=0
\end{array}
$$

The nondimensionalized system of Equations (16) and (17) are subject to non-dimensionalized boundary conditions Equations (18) and (19).

$$
\begin{array}{rlrl}
U(-1) & =0 & U(1) & =0 \\
\theta(-1) & =0 & \theta(1) & =1
\end{array}
$$

The viscous dissipation effects and magnetic field effects on temperature are taken into account for this one dimensional problem. The equations are first discretized utilizing GDQM. Then the resulting system of algebraic equations are solved by Newton-Raphson Algorithm.

\subsection{Generalized Differential Quadrature Method (GDQM)}

To solve the nonlinear coupled differential equations in Equations (16) and (17), subject to boundary conditions in Equations (18) and (19), GDQM method is used for discretization. Differential Quadrature, inspired by integral quadrature, is a powerful technique to approximate the derivatives of a function at a point as a weighted linear sum of all the functional values at discrete points along the derivation direction. DQM states that the $n$-th order derivative of a single variable function $u(y)$ can be approximated as Equation (20).

$$
u^{(n)}\left(y_{i}\right)=\sum_{j=1}^{N} c_{i j}^{(n)} u\left(y_{j}\right), \quad i=1,2, \ldots, N
$$

where $c_{i j}^{(n)}$ are the weighting coefficients for the $n^{\text {th }}$ order derivative, and $N$ is the total number of sampling points of the grid distribution. The key paradigm in DQM lies in the calculation of weighting coefficients. This is also the main reason that the method is not widely used till last decade. The founder of the method Bellmann [52], proposed two methods for calculation of first order coefficient. First method allows to choose the coordinates of the grid points arbitrarily but is restricted to use of a small number of grid point, i.e., 13 grid points due to ill-conditioned Vandermorde matrix. The second method utilizes a simple algebraic formulation, but the selection of grid point coordinates are the roots of the shifted Legendre polynomial, not arbitrary. These drawbacks limited the use of method until a major breakthrough is attained by Shu and Richards [53] where all the methods available are generalized under the analysis of a high order polynomial approximation and analysis of 
a linear vector space. Their approach lets the user calculate the second and higher order weighting coefficients by a recurrence relationship with arbitrary choice of grid points [54]. Equations (20) and (21) describes methodology to calculate the weighting coefficients.

$$
c_{i j}^{(n)}= \begin{cases}\frac{M^{(1)}\left(y_{i}\right)}{\left(y_{i}-y_{j}\right) M^{(1)}\left(y_{j}\right)} & \text { for } i \neq j \\ -\sum_{j=1, j \neq i}^{N} c_{i j}^{(1)} & \text { for } i=j\end{cases}
$$

where $M^{(1)}$ can be written as Equation (22),

$$
M^{(1)}\left(y_{k}\right)=\prod_{j=1, j \neq k}^{N}\left(y_{k}-y_{j}\right)
$$

To solve the weighting coefficients of higher order derivatives, following recurrence relations, Equations (22) and (23), are utilized.

$$
c_{i j}^{(n)}= \begin{cases}n\left(c_{i i}^{(n-1)} c_{i j}^{(1)}-\frac{c_{i j}^{(n-1)}}{y_{i}-y_{j}}\right) & \text { for } i \neq j \\ -\sum_{j=1, j \neq i}^{N} c_{i j}^{(n)} & \text { for } i=j\end{cases}
$$

The grid distribution is chosen as Chebyshev-Gauss-Lobatto grid, which is denser at the places closer to the boundaries, and it gives much better results when compared to uniform grid distribution. The mathematical description giving the coordinates of the grids can be found in Equation (24).

$$
y_{i}=\cos \left(\frac{i-1}{N-1} \pi\right)
$$

\subsection{Entropy Generation}

Designing optimal engineering systems is a key goal in energy efficiency. To predict the performance of the processes, second law of thermodynamics has been applied for years. However, Bejan introduced the idea of entropy generation minimization to optimize heat exchange system. The convection process in a channel is inherently irreversible which causes entropy generation. Local volumetric entropy generation rate formula for a viscous incompressible conducting fluid in the presence of magnetic field is given by [55] as Equation (25), indicating each term's irreversibility source. Irreversibility has been referred since it gives an idea for the loss of available work of the system. Irreversibility is mentioned to point to different sources of entropy generation (magnetic field, viscous dissipation, heat transfer), since entropy is produced in irreversible processes.

$$
E_{G}=\underbrace{\frac{k}{T_{2}^{2}}\left(\frac{d T}{d y}\right)^{2}}_{\text {heat_transfer }}+\underbrace{\frac{\mu}{T_{2}}\left(\frac{d u}{d y}\right)^{2}}_{\text {viscous_dissipation }}+\underbrace{\frac{\sigma B_{0}^{2}}{T_{2}} u^{2}}_{\text {magnetic_field }}
$$

The last term on the right hand side of Equation (25) is the entropy generation due to magnetic field. Evaluating non-dimensional parameters given in Equations (9)-(14), local entropy generation rate is defined as in Equation (26). 


$$
N_{S}=\frac{T_{2}^{2} h^{2} E_{G}}{k\left(T_{1}-T_{2}\right)^{2}}=\left(\frac{d \theta}{d Y}\right)^{2}+\frac{B r}{\Omega}\left(e^{-\varepsilon \theta}\left(\frac{d U}{d Y}\right)^{2}+H a U^{2}\right)
$$

Here $\Omega$ and $B r$ stand for the temperature difference parameter and Brinkmann number respectively and can be given as $\Omega=\frac{\left(T_{1}-T_{2}\right)}{T_{2}}, B r=E c \operatorname{Pr}$. To show the dominance of irreversibility due to heat transfer with respect to the combined effect of fluid friction and magnetic fields, Bejan number is introduced as follows

$$
B e=\frac{N_{\text {heat }}}{N_{S}}=\frac{\left(\frac{d \theta}{d Y}\right)^{2}}{\left(\frac{d \theta}{d Y}\right)^{2}+\frac{B r}{\Omega}\left(e^{-\varepsilon \theta}\left(\frac{d U}{d Y}\right)^{2}+H a U^{2}\right)}
$$

Be number ranges between 0 and 1 with a meaning of dominance of irreversibility due to heat transfer dominates for the values closer to 1 , and the combination of fluid friction and magnetic field for the values closer to 0 .

\section{Simulations and Results}

In this section, simulations are carried out for a variety of parameters and solved via GDQM $\&$ NR methods. The velocity and temperature distributions, local entropy generation and total entropy generation are investigated with respect to key system parameters, such as magnetic field angle, inclination of the channel, Ha number and nanoparticle volume fraction. The codes are implemented in MATLAB environment.

Since the numeric solution is designed in-house, the need to verify its validity, a simpler geometry is refered where numerical results are easily available in the literature and simplifies to an analytical solution under some conditions. This study adopts a suction-injuction channel flow without nanofluids. The results show that DQM tool implemented in-house gives even more accurate results then Runge-Kutta solutions given in [45] for the specific case where an analytical solution is also available for comparison [56]. Furthermore, another strong feature of DQM is observed, ability to give relatively accurate solutions for few number of grids. Further verification has been proceed with a comparison with the literature for trends of change of velocity and temperature profiles while parameters of the flow is changed such as Ha number.

This problem is designed such that the user of the system would have more parameters to configure the flow. One of the main controllable parameter is angle of the magnetic field. Throughout this study, the direction of the magnetic field is not assumed to be perpendicular to the channel walls while the inclination of the channel is changing. This configuration might have been helpful to have an insight for the applications where inclination of the channel could not be changed but the magnetic field angle could be changed.

First of all, effect of magnetic field magnitude (discarding the direction) on the flow is investigated by changing the dimensionless $\mathrm{Ha}$ number but keeping the magnetic field direction constant. Considered parameters for the simulation given in Figures 2 and 3 are $P=1 ; B r=0.1 ; R a=10$; $\operatorname{Pr}=6.7 ; \alpha=10^{\circ} ; \phi=30^{\circ}$. Figures 2 and 3 also shows the effect of nanoparticle volume fraction on velocity and temperature profiles. By letting more particles to the system or discarding some, the flow can be supervised. In the simulations, the particle volume fraction is changed between $\psi=0-0.06$ from where $\psi=0$ corresponding to the pure base fluid case without nanoparticles. The velocity decreases with an increase in volume fraction of nanofluid.

Figure 3 shows the effect of Hartmann number on the velocity profile. It is clear that, increasing the value of Ha have a tendency to slow down the fluid motion because of the presence of the transverse magnetic field. Transverse magnetic field creates a resistive force similar to the drag force, which acts in the opposite direction of the fluid motion. The velocity of the fluid decreases with resistive force and it is clear that the applied magnetic field has a retarding effect on the flow field. This is also the case for 
fluids without nanoparticles but results show that for a concentration of $\psi=0.06$, the magnetic field braking effect increases when compared to the case of no particles, i.e., $\psi=0$.

Figure 2 shows the temperature variation is almost linear with respect to non-dimensionalized channel width and decrease with increasing magnetic field intensity. The fluid temperature decreases increasing the value of Ha within the channel due to increasing magnetic field intensity. This behavior is attributed to decrease the fluid velocity due to the magnetic field.

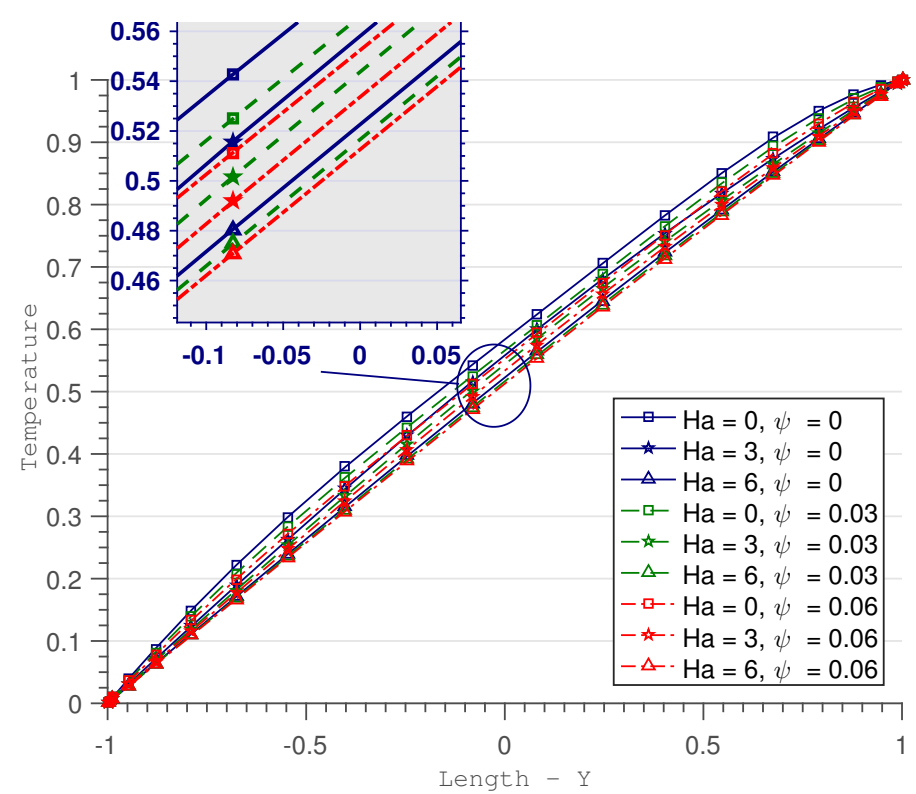

Figure 2. Nondimensionalized temperature change with channel width for a variety of magnetic field intensity and solid volume fraction.

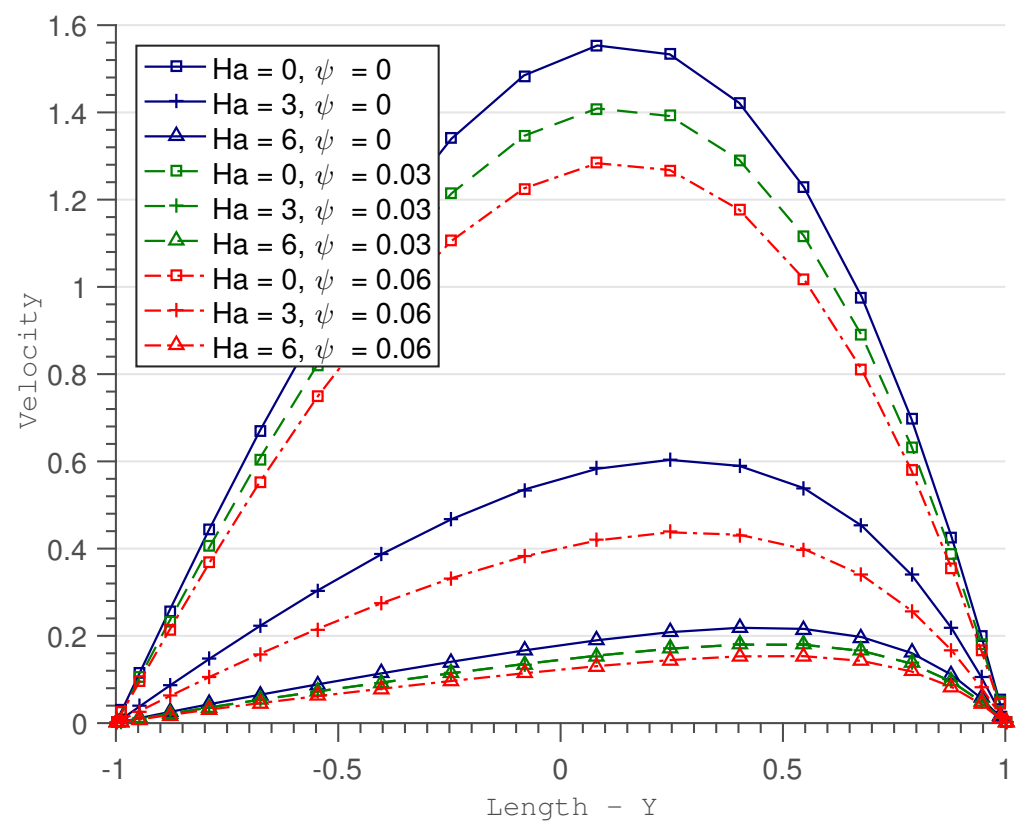

Figure 3. Nondimensionalized velocity change with channel width for a variety of magnetic field intensity and solid volume fraction.

As mentioned above, the main parameter to be utilized in order to achieve more dominance over the behavior of the flow is the magnetic field angle $(\alpha)$. To further differentiate the effect of magnetic field and gravitation over the flow, the magnetic field angle is offered to be independent from the 
angle of the channel. This could be a good modeling approach for the problems where the channel has a fixed inclination while the magnetic field can be directed independently. An example case could be an medical application where the veins should not be moved while the magnetic field applied from outside could be changed in favor of the application.

Figure 4 shows the behavior of the velocity along the channel for a variety of magnetic field and channel inclination values for $\psi=0.06$ and $\mathrm{Ha}=2$. The rise in the inclination causing an increase in the flow velocity is observed, obviously matching the common sense. The velocity profiles are unsymmetrical about the centerline of the channel due to inclination angle as expected. It also shows that when the inclination of the magnetic field increased, the flow velocity decreases. A key point to realize is that the flow velocity can be decreased to a level less then even a higher inclination of the channel velocity profile by changing the magnetic field angle less then $45^{\circ}$. This can be seen clearly from the curves representing $\alpha=0^{\circ}, \phi=10$ and $\alpha=45^{\circ}, \phi=20^{\circ}$ in Figure 4. This gives the user of the channel a good dominance to control the flow as is intended. A second point in Figure 4 is that for higher values of magnetic field angle, the less change will be observed in the velocity change corresponding to a change in magnetic field angle. This can be observed by checking the same color curves, and realize that the difference of flow velocity suppression is higher in the change from $\alpha=0^{\circ}$ to $\alpha=45^{\circ}$ compared to the change in $\alpha=45^{\circ}$ to $\alpha=90^{\circ}$. The flow velocity supression in higher magnetic field inclinations also decreases for higher channel inclination. This can be seen by observing the decrease in velocity for $\alpha=45^{\circ}$ to $\alpha=90^{\circ}$ for $\phi=20^{\circ}$ is less than the decrease in velocity for $\alpha=45^{\circ}$ to $\alpha=90^{\circ}$ for $\phi=10^{\circ}$. Another result is that these behaviour does not change for different nano-particle volume fractions $\psi$ but not showed here due to page limitations.

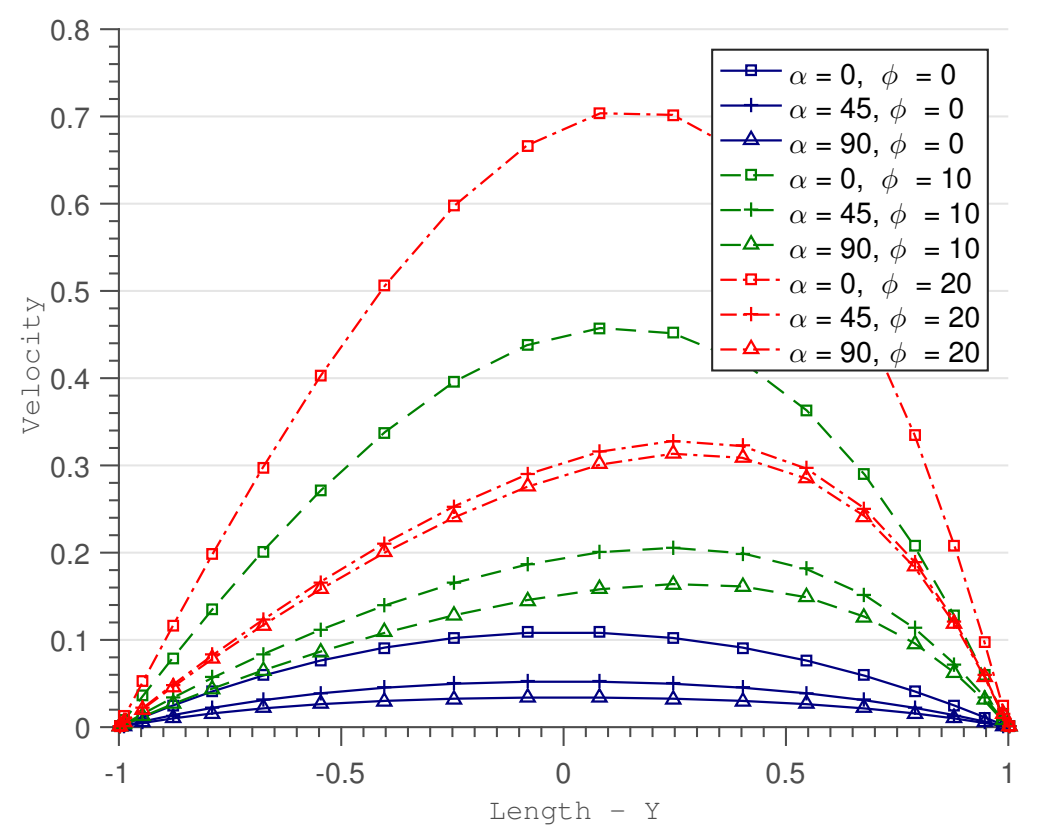

Figure 4. Nondimensionalized velocity with respect to magnetic field angle $\alpha$ and channel inclination $\phi$.

Figure 5 is a broader look at the trend of the effect of $\alpha$ and $\phi$ on flow velocity, by showing 3D figures representing the change of flow velocity with respect to inclination of the channel $\phi$ for four different magnetic field angle $\alpha$. The results discussed until now can be seen in this figure as well as a trend in change in shape of the surface for different values of magnetic field angles $\alpha$. 

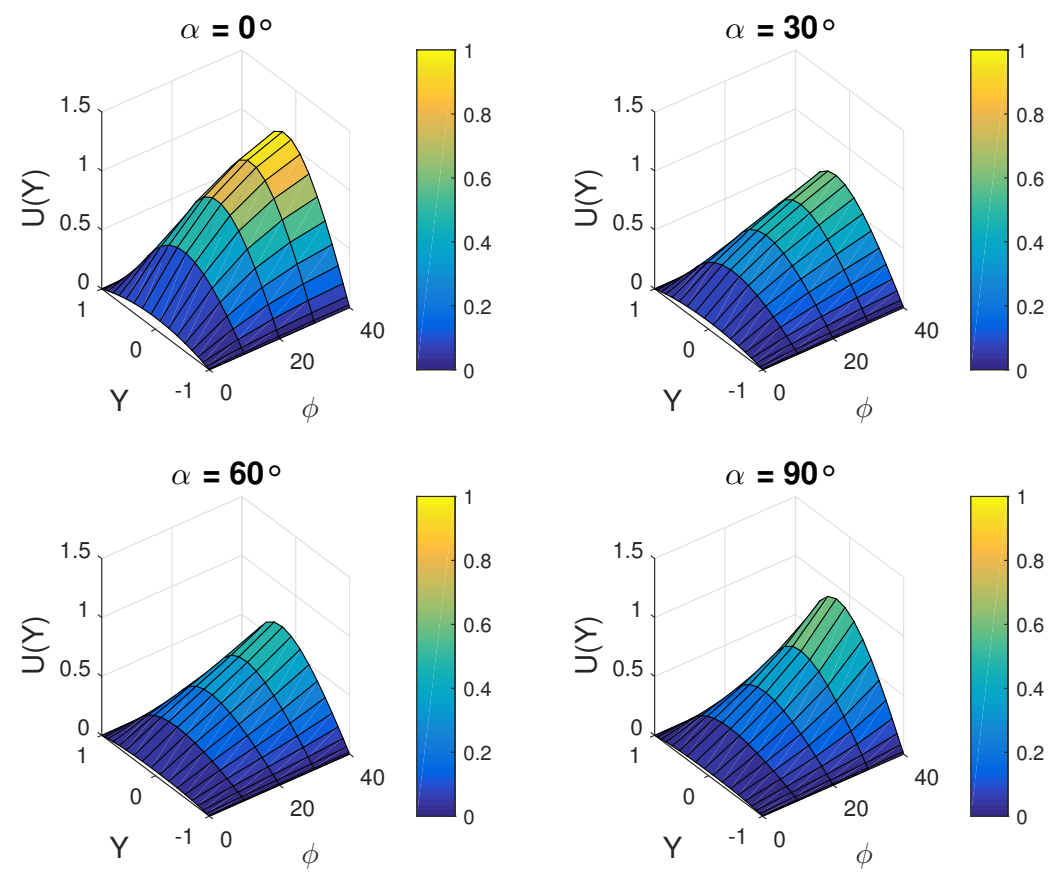

Figure 5. 3D flow behaviour surface for nondimensionalized velocity with respect to channel inclination $\phi$ for four different magnetic field angle $\alpha$.

Engineering system design is a complicated process and most of the times requires comprise from one property or another. A wise choice to tune in between favorable properties, there are various optimization methods. One of them is to calculate and minimize the entropy generation. The influences of the different parameters on entropy generation within the channel are presented in Figures 6-9. It is seen from Figures that the entropy generation depends on magnetic field angle, channel inclination. Using the local non-dimensional entropy generation rate formula for nanofluid in the presence of magnetic field is given in Equation (26), local entropy generation for a variety of $\alpha$ and $\phi$ for $H a=2, \psi=0.06$ and given in Figure 6 . It shows that the entropy generation trend changes heavily for different channel inclination angle $\phi$. A change in magnetic field angle $\alpha$ may also effect the entropy generation abruptly. When the magnetic field angle is increased, a decrease in entropy generation is observed in Figure 6. But this effect gets nearly negligible for higher values of magnetic field angle. This can be checked by investigating the distance in between the local entropy generation curves for $\alpha=0-45^{\circ}$ and $\alpha=45-90^{\circ}$. Furthermore, addition of nanoparticles decrease the local entropy generation but does not change the effect of $\alpha$ and $\phi$ on the local entropy generation. Here only the trend for $\psi=0.06$ is given but in the course of the study, the effect of $\alpha$ and $\phi$ on the local entropy generation is investigated for $\psi=0,0.03,0.06$ but not all given due to the page constraints.

The influences of the different parameters on entropy generation within the channel are presented in Figures 6-9. It is seen from Figs that the entropy generation number NS depends on magnetic field angle, channel inclination.

To gain further insight about the behavior of local entropy generation as a function of magnetic field angle and channel inclination, four 3D graphes are presented as Figure 7. In this simulation $H a=2$ and $\psi=0.06$. These four surfaces in Figure 7 present the change of local entropy generation with magnetic field angle $\alpha$ for four different channel inclinations $\phi=0^{\circ}, 20^{\circ}, 30^{\circ}, 40^{\circ}$. It can be seen from the upper left surface corresponding to $\phi=0^{\circ}$, the change in magnetic field angle $\alpha$ does not help much to decrease the local entropy generation. But for channel inclination $\phi=20^{\circ}$, local entropy generation is decreased for higher values of $\alpha$. For higher values of $\phi, \phi=30^{\circ}, 40^{\circ}$, an increase in $\alpha$ first results in a decrease in local entropy generation. But after some specific value, the local entropy generation starts to increase for increasing $\alpha$. This can be seen also from Figure 8 , where 
local entropy generation is averaged along the width of channel and no longer dependent on the location, namely total entropy generation. Those result means that magnetic field angle can be used to decrease local entropy generation but depending on the inclination of the channel, this might be the contrary. This means that the user can utilize these behavior to optimize the system depending on the perquisites of the problem. Another result observed is that this behavior is not changed for nanoparticle volume fraction $\psi$. But for larger $\psi$, a general decreasing effect is seen in the simulations for all values of $\alpha$ and $\phi$.

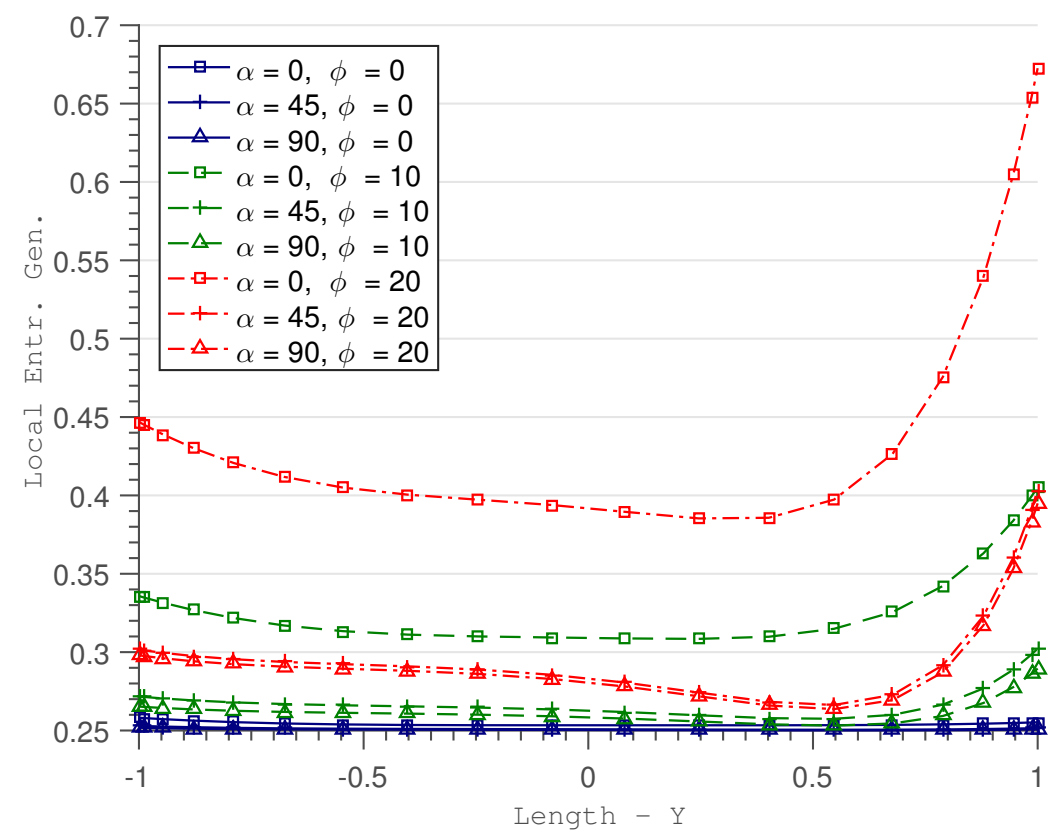

Figure 6. Local entropy generation as a function of channel width for specific magnetic field angle $\alpha$ and channel inclination $\phi$.
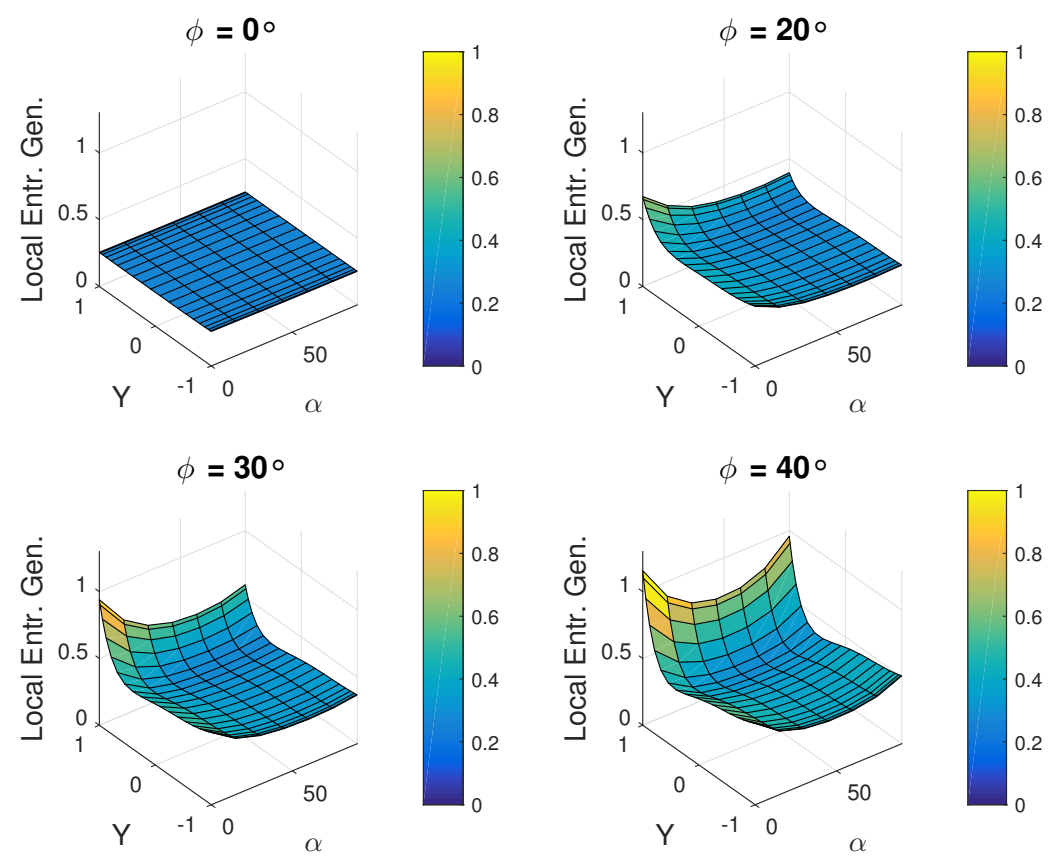

Figure 7. Local entropy generation as a function of magnetic field angle and channel width for four different channel inclinations $\phi=0^{\circ}, 20^{\circ}, 30^{\circ}, 40^{\circ}$. 


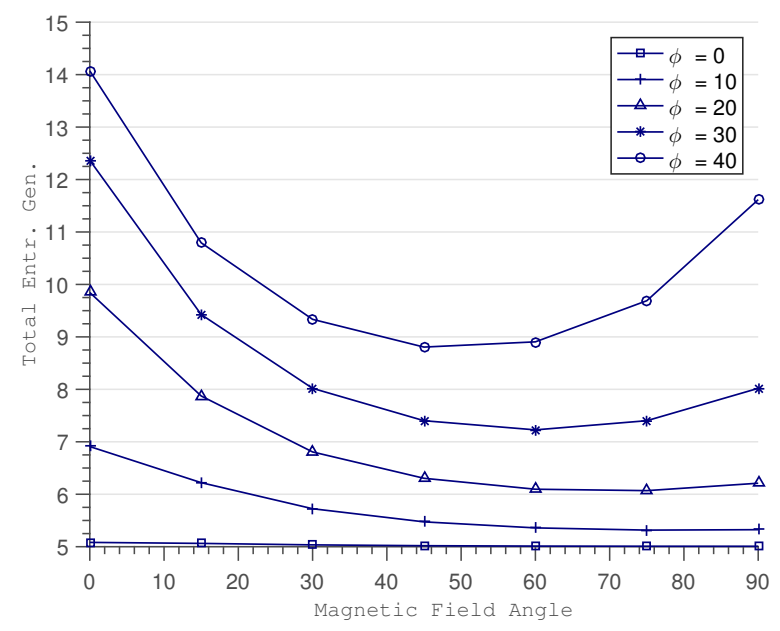

Figure 8. Total entropy generation as a function of magnetic field angle $\alpha$ for a variety of channel inclination $\phi$.

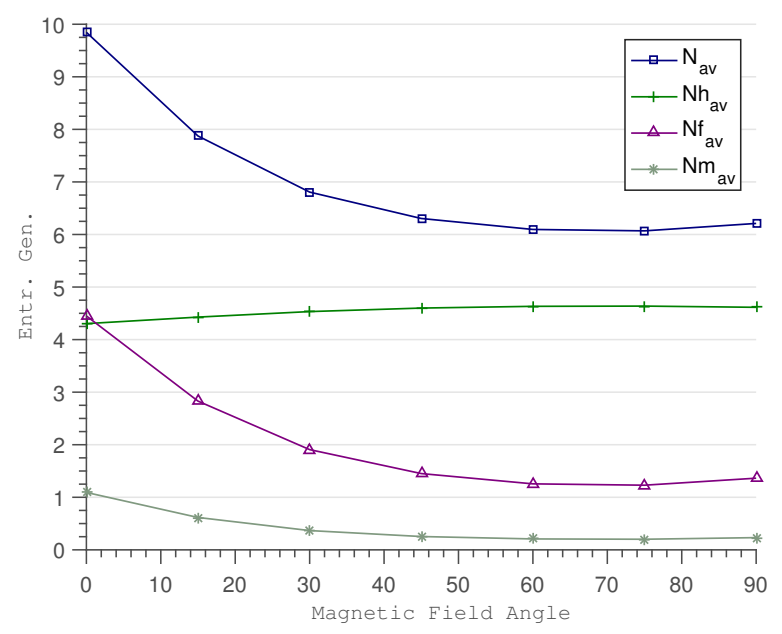

Figure 9. Total entropy generation due to its terms; heat transfer term, viscous dissipation term, magnetic field term as a function of magnetic field angle $\alpha$ for channel inclination $\phi=20$.

A further investigation given in Figure 9 on the total entropy generation concerns the visualization of entropy generation terms separately. As seen from Equation (25), the entropy generations is comprised of entropy generation due to heat transfer irreversibility, entropy generation due to fluid friction irreversibility and entropy generation due to magnetic field. Figure 9 shows that the decrease in total entropy generation via an increase in magnetic field angle belongs to viscous dissipation and magnetic field terms of entropy generation. The entropy generation term due to heat transfer irreversibility remains nearly unchanged. To achieve a further decrease in entropy generation, this terms might be handled with a different controllable parameter.

\section{Discussion}

In the present work, the effects of magnetic field orientation angle and channel inclination angle are separately investigated, when the channel filled with ferrofluid, on the entropy generation. And for such a channel system, related parameters investigated to produce minimum entropy generation case. Therefore, the effect of magnetic field magnitude, channel inclination angle and the volume fraction on the velocity and temperature distributions. The viscous dissipations and buoyancy effects are included in the governing equations. Derived governing equations are non-dimensionalized by using physically appropriate parameters. 
Equations for flow and thermal fields are discretized using GDQM, a new computationally efficient tool giving fairly accurate results for even very few grid points. The discretized system of equations are solved simultaneously utilizing Runge-Kutta scheme. An increase in magnetic field density suppresses the flow field significantly, and these effects get stronger as the volume fraction of nanoparticles in nanofluids increases. The velocity and temperature increases as the inclination of the channel rises but this tendency diminishes with larger magnetic field intensity or volume fraction of nanofluids. The velocity decreases with an increase in volume fraction of nanoparticles. When magnetic field gets stronger, the volume fraction dependence of the velocity also increases and gets more dependent to temperature distribution. Influence of a change in particle volume fraction on temperature distribution is minor and diminished with increased magnetic field magnitude. The entropy generation decreases with increasing magnetic field angle for smaller values of channel inclination. For higher values of channel inclination, with an increase in magnetic field angle, the entropy generation first decreases and then increases. The minimum entropy generation is observed around when the magnetic field angle is perpendicular to the channel. Thus, to further optimize the system by managing velocity and temperature distributions, an multi-objective optimization method should be utilized to serve the users needs.

Author Contributions: All authors have read and approved the final manuscript.

Conflicts of Interest: The authors declare no conflict of interest.

\section{Abbreviations}

The following abbreviations are used in this manuscript:

$B_{0} \quad$ magnetic field intensity

$c_{p} \quad$ specific heat $(\mathrm{J} / \mathrm{K})$

$H$ channel diameter $(\mathrm{m})$

$N \quad$ number of grids

$g$ gravitational acceleration $\left(\mathrm{m} / \mathrm{s}^{2}\right)$

$k$ thermal conductivity $(\mathrm{W} / \mathrm{mK})$

$\alpha \quad$ magnetic field orientation angle (rad)

$\phi \quad$ inclination angle (rad)

$\alpha_{f} \quad$ thermal diffusivity $\left(\mathrm{m} / \mathrm{s}^{2}\right)$

$\psi \quad$ solid volume fraction

$\mu \quad$ dynamic viscosiy $\left(\mathrm{m} / \mathrm{s}^{2}\right)$

$v \quad$ kinematics viscosity $\left(\mathrm{m} / \mathrm{s}^{2}\right)$

$\sigma \quad$ electrical conductivity

$\mathrm{Ha}$ Hartmann number

$\mathrm{Ra}$ Rayleigh number

Ec Eckert number

Pr Prantl number

$\mathrm{Br} \quad$ Brinkman number

$u \quad \mathrm{x}$ component of velocity vector $(\mathrm{m} / \mathrm{s})$

$U$ dimensionless $\mathrm{x}$ component of velocity vector

$T$ temperature (K)

$\theta \quad$ dimensionless temperature

$x, y \quad$ cartesian coordinates $(\mathrm{m})$

$X, Y$ dimensionless cartesian coordinates

$f \quad$ fluid

$p \quad$ nanoparticle

$n f$ nanofluid 


\section{References}

1. Maxwell, J.C. A Treatise on Electricity and Magnetism; Clarendon: Oxford, UK, 1904.

2. Choi, S.U.S.; Eastman, J.A. Enhancing thermal conductivity of fluids with nanoparticles. In Proceedings of the ASME International Mechanical Engineering Congress \& Exposition, San Francisco, CA, USA, 12-17 November 1995.

3. Eastman, J.; Choi, U.; Li, S.; Thompson, L.; Lee, S. Enhanced thermal conductivity through the development of nanofluids. MRS Online Proc. Libr. Arch. 1996, 457, doi:10.1557/PROC-457-3.

4. Liu, M.S.; Lin, M.C.; Huang, I.T.; Wang, C.C. Enhancement of thermal conductivity with CuO for nanofluids. Chem. Eng. Technol. 2006, 29, 72-77.

5. Hwang, Y.; Park, H.; Lee, J.; Jung, W. Thermal conductivity and lubrication characteristics of nanofluids. Curr. Appl. Phys. 2006, 6, e67-e71.

6. Yu, W.; Xie, H.; Chen, L.; Li, Y. Investigation of thermal conductivity and viscosity of ethylene glycol based ZnO nanofluid. Thermochim. Acta 2009, 491, 92-96.

7. Mintsa, H.A.; Roy, G.; Nguyen, C.T.; Doucet, D. New temperature dependent thermal conductivity data for water-based nanofluids. Int. J. Therm. Sci. 2009, 48, 363-371.

8. Verma, P.; Chaturvedi, P.; Rawat, J.S.; Kumar, M.; Pal, S.; Bal, M.; Rawal, D.; Vyas, H.P.; Ghosal, P.; Bhatnagar, P. Elimination of current non-uniformity in carbon nanotube field emitters. J. Mater. Sci. Mater. Electron. 2007, 18, 677-680.

9. Xu, J.; Zhang, J.; Du, Y.; Zhang, X.; Li, Y. Ultrasonic velocity and attenuation in nano-structured Zn materials. Mater. Lett. 1996, 29, 131-134.

10. Eastman, J.A.; Choi, S.; Li, S.; Yu, W.; Thompson, L. Anomalously increased effective thermal conductivities of ethylene glycol-based nanofluids containing copper nanoparticles. Appl. Phys. Lett. 2001, 78, 718-720.

11. Bondareva, N.; Sheremet, M.A.; Pop, I. Magnetic field effect on the unsteady natural convection in a right-angle trapezoidal cavity filled with a nanofluid: Buongiorno's mathematical model. Int. J. Numer. Methods Heat Fluid Flow 2015, 25, 1924-1946.

12. Sheremet, M.A.; Pop, I. Natural convection in a wavy porous cavity with sinusoidal temperature distributions on both side walls filled with a nanofluid: Buongiorno's mathematical model. J. Heat Transf. 2015, 137, 072601.

13. Baskaya, E.; Fidanoglu, M.; Komurgoz, G.; Ozkol, I. Investigation of MHD natural convection flow exposed to constant magnetic field via generalized differential quadrature method. In Proceedings of the ASME 12th Biennial Conference on Engineering Systems Design and Analysis, Copenhagen, Denmark, 25-27 July 2014.

14. Jang, S.P.; Choi, S. Effects of Various Parameters on Nanofluid Thermal Conductivity. J. Heat Transf. 2006, 129, 617-623.

15. Nacev, A. Magnetic Drug Targeting: Developing the Basics. Ph.D. Thesis, The University of Maryland, College Park, MD, USA, 2013.

16. Arruebo, M.; Fernandez-Pacheco, R.; Ibarra, M.R.; Santamaria, J. Magnetic nanoparticles for drug delivery. Nano Today 2007, 2, 22-32.

17. Pankhurst, Q.A.; Connolly, J.; Jones, S.K.; Obson, J. Applications of magnetic nanoparticles in biomedicine. J. Phys. Appl. Phys. 2003, 36, R167.

18. Pankhurst, Q.A.; Thanh, N.K.; Jones, S.K.; Obson, J. Progress in applications of magnetic nanoparticles in biomedicine. J. Phys. Appl. Phys. 2009, 42, 224001.

19. Lubbe, A.S.; Bergemann, C.; Riess, H.; Schriever, F.; Reichardt, P.; Possinger, K.; Matthias, M.; Dorken, B.; Herrmann, F.; Gurtler, R.; et al. Clinical experiences with magnetic drug targeting: A phase I study with 4-epidoxorubicin in 14 patients with advanced solid tumors. Cancer Res. 1996, 56, 4686-4693.

20. Obson, J. Magnetic micro- and nano-particle-based targeting for drug and gene delivery. Nanomedicine 2006, 1, 31-37.

21. Sheremet, M.A.; Oztop, H.F.; Pop, I.; Abu-Hamdeh, N. Analysis of entropy generation in natural convection of nanofluid inside a square cavity having hot solid block: Tiwari and Das model. Entropy 2015, 18, 9.

22. Carnot, N. Reflexions Sur la Puissance Motrice du feu (In France); Bachelier: France, Paris, 1824.

23. Clausius, R. On a modified form of the second principal theorem of mechanical theory. Ann. Phys. 1854, 169, 481-506.

24. Clausius, R. On various forms of the principal equations of mechanical theory. Ann. Phys. 1865, 201, 353-400.

25. Bejan, A. Second law analysis in heat transfer. Energy Int. 1980, 5, 721-732. 
26. Bejan, A. Entropy Generation Through Heat and Fluid Flow; Wiley: New York, NY, USA, 1982.

27. Bejan, A. Second-law analysis in heat transfer and thermal design. Adv. Heat Transf. 1982, 15, 1-58.

28. Bejan, A. Entropy Generation Minimization; CRC Press: New York, NY, USA, 1995.

29. Bejan, A. A study of entropy generation in fundamental convective heat transfer. J. Heat Transf. 1979, 101, 718-725.

30. Bejan, A.; Tsatsaronis, G.; Moran, M. Thermal Design and Optimization; John Wiley \& Sons: New York, NY, USA, 1996.

31. Roy, M.; Basak, T.; Roy, S.; Pop, I. Analysis of Entropy Generation for Mixed Convection in a Square Cavity for Various Thermal Boundary Conditions. Numer. Heat Transf. Part A Appl. 2015, 68, 44-74.

32. Bhardwaj, S.; Dalal, A. Effect of Undulations on the Natural Convection Heat Transfer and Entropy Generations Inside a Porous Right-Angled Triangular Enclosure. Numer. Heat Transf. Part A Appl. 2015, 67, 972-991.

33. Yang, Y.T.; Wang, Y.H.; Yi-Hsien, H.; Huang, B.Y. Numerical Optimization for Nanofluid Flow in Microchannels using Entropy Generation Minimization. Numer. Heat Transf. Part A Appl. 2015, 67, 571-588.

34. Komurgoz, G.; Arikoglu, A.; Turker, E.; Ozkol, I. Second-Law Analysis for an Inclined Channel Containing Porous-Clear Fluid Layers by Using the Differential Transform Method. Numer. Heat Transf. Part A Appl. 2010, 57, 603-623.

35. Salas, H.; Cuevas, S.; de Haro, M.L. Entropy Generation Analysis of Magnetohydrodynamic Induction Devices. J. Phys. D Appl. Phys. 1999, 32, 2605-2608.

36. Mahmud, S.; Tasnim, S.H.; Mamun, M.A.H. Thermodynamic Analysis of Mixed Convection in a Channel with Transverse Hydromagnetic Effect. Int. J. Therm. Sci. 2003, 42, 731-740.

37. Chauhan, D.S.; Kumar, V. Heat transfer and entropy generation during compressible fluid flow in a channel partially filled with porous medium. Int. J. Energy Technol. 2011, 3, 1-10.

38. Tasnim, S.; Mahmud, S.; Mamum, M. Entropy generation in a porous channel with hydromagetic effect. Int. J. Exergy 2002, 3, 300-308.

39. Eegunjobi, A.S.; Makinde, O.D. Combined effect of buoyancy force and Navier slip on entropy generation in a vertical porous channel. Entropy 2012, 14, 1028-1044.

40. El Jery, A.; Hidouri, N.; Magherbi, M.; Brahim, A.B. Effect of an External Oriented Magnetic Field on Entropy Generation in Natural Convection. Entropy 2010, 12, 1391-1417.

41. Dwivedi, R.; Singh, S.P.; Singh, B.B. Analysis of incompressible viscous laminar flow through a channel filled with porous media. Int. J. Stab. Fluid Mech. 2010, 1, 127-134.

42. Makinde, O.D.; Chinyoka, T. Numerical investigation of buoyancy effects on hydromagnetic unsteady flow through a porous channel with suction/injection. Mech. Sci. Technol. 2013, 27, 1557-1568.

43. Cramer, K.; Pai, S. Magnetofluiddynamics for Engineers and Applied Plysicists; McGraw Hill Book Company: New York, NY, USA, 1973.

44. Seth, G.S.; Ansari, M.S.; Nandkeolyar, R. Unsteady Hydromagnetic Couette Flow within a porous Channel. Tamkang J. Sci. Eng. 2011, 14, 7-14.

45. Eegunjobi, A.S.; Makinde, O.D. Entropy Generation Analysis in a Variable Viscosity MHD Channel Flow with Permeable Walls and Convective Heating. Math. Probl. Eng. 2013, 2013, 630798.

46. Das, S.; Jana, R.N. Entropy Generation in MHD Porous Channel Flow Under Constant Pressure Gradient. Appl. Math. Phys. 2013, 1, 78-89.

47. Hayat, T.; Bibi, S.; Rafiq, M.; Alsaedi, A.; Abbasi, F. Effect of an inclined magnetic field on peristaltic flow of Williamson fluid in an inclined channel with convective conditions. J. Magn. Magn. Mater. 2016, 401, 733-745.

48. Mehrez, Z.; El Cafsi, A.; Belghith, A.; Le Quéré, P. MHD effects on heat transfer and entropy generation of nanofluid flow in an open cavity. J. Magn. Magn. Mater. 2015, 374, 214-224.

49. Cimpean, D.S.; Pop, I. Fully developed mixed convection flow of a nanofluid through an inclined channel filled with a porous medium. Int. J. Heat Mass Transf. 2012, 55, 907-914.

50. You, X.C.; Xu, H.; Pop, I. Analysis of Fully Developed Opposing Mixed Convection Flow in an Inclined Channel Filled by a Nanofluid. J. Heat Transf. 2014, 136, 124502.

51. Brinkmann, H.C. The Viscosity of Concentrated Suspensions and Solutions. J. Chem. Phys 1952, 20, 571-581.

52. Bellman, R.E.; Casti, J. Differential Quadrature and Long Term Integration. J. Math. Anal. Appl. 1971, 34, 235-238. 
53. Shu, C.; Richards, B.E. High Resolution of Natural Convection in a Square Cavity by Generalized Differential Quadrature. In Proceedings of the 3rd Conference on Advances in Numerical Methods in Engineering, Swansea, UK, 1990.

54. Shu, C. Differential Quadrature and Its Applications in Engineering; Springer-Verlag: London, UK, 2000.

55. Woods, L.C. Thermodynamics of Fluid Systems; Oxford University Press: Oxford, UK, 1975.

56. Başkaya, E.; Kömürgöz, G.; Özkol, I. Analysis of Variable Viscosity Channel Flow under Constant Magnetic Field via Generalized Differential Quadrature Method. In Advanced Materials Research; Trans Tech Publications: Zurich, Switzerland, 2014. article distributed under the terms and conditions of the Creative Commons Attribution (CC BY) license (http://creativecommons.org/licenses/by/4.0/). 